FECHA DE

PUBLICACIÓN: 10-05-2021

FECHA DE ACEPTACIÓN: 20-04-2021

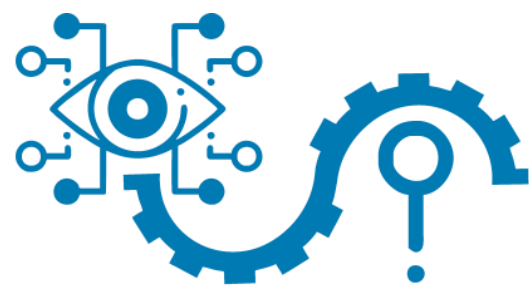

GnasisWisdam
REVISTA DE INVESTIGACIÓN E INNOVACIÓN CIENTÍFICA Y TECNOLÓGICA

Artículo original

Volumen 1, Número 2, Mayo - Agosto 2021

\title{
EL MÉTODO POLYA COMO ESTRATEGIA EN LA RESOLUCIÓN DE PROBLEMAS ARITMÉTICOS DE ENUNCIADO VERBAL EN ESTUDIANTES DE PRIMARIA
}

\section{THE POLYA METHOD AS A STRATEGY IN THE RESOLUTION OF ARITHMETIC PROBLEMS OF VERBAL STATEMENT IN ELEMENTARY STUDENTS}

\author{
Autores \\ Lino, Vilca Mamani ORCID \\ Universidad Nacional del Altiplano Puno \\ Bertha, Hancco Zela ORCID \\ Universidad Nacional Intercultural de la Amazonía \\ Benito, Navarro Quisocala ORCID \\ Universidad Nacional de San Agustin \\ Mirian, Loza Cauna ORCID \\ Instituto de Educación Superior Pedagógico Público Puno
}

\section{Resumen}

El Método Polya como estrategia en la resolución de problemas aritméticos de enunciado verbal en estudiantes de primaria, se realiza para alcanzar el objetivo de determinar el nivel de logro de resolución de problemas aritméticos de enunciado verbal (PAEV) en estudiantes de cuarto grado de la IEP N 71001 Almirante Miguel Grau de la ciudad de Puno en el año 2017, debido a que se observó que en la Institución Educativa en mención, los estudiantes de cuarto grado tenían dificultades en la resolución de problemas matemáticos, es por ello que aplicando un diseño de investigación cuasi experimental a una muestra de 64 niños y niñas, y utilizando como instrumento una prueba de salida, se pudo llegar a las siguientes resultados: El método Polya, permite mejorar los niveles de logro en la resolución de problemas aritméticos de enunciado verbal en los estudiantes investigados, pues este método facilita desarrollar habilidades de resolución de problemas aditivos y multiplicativos utilizando sus propiedades en cada caso, y en forma general, los estudiantes del grupo experimental alcanzaron obtener una media aritmética de 14,89 puntos, mientras que en el grupo de control sólo fue de 12,39 puntos.

Palabras clave: Problemas de cambio, combinación, comparación, igualación.

\begin{abstract}
The Polya Method as a strategy in solving arithmetic verbal enunciation problems in elementary school students, is carried out to achieve the objective of determining the level of achievement of
\end{abstract}




\section{Bnosis Wisdom}

solving arithmetic verbal enunciation problems (PAEV) in fourth grade students of the IEP $\mathrm{N}^{\circ}$ 71001 Almirante Miguel Grau from the city of Puno in 2017, because it was observed that in the Educational Institution in question, fourth grade students had difficulties in solving mathematical problems, that is why applying a design of Quasi-experimental research on a sample of 64 boys and girls, and using an exit test as an instrument, the following results could be reached: The Polya method, allows to improve the levels of achievement in solving arithmetic problems of verbal enunciation in the investigated students, as this method facilitates the development of additive and multiplicative problem solving skills using their properties in each case, and in general, the students in the experimental group achieved an arithmetic mean of 14.89 points, while in the control group it was only 12.39 points.

Keywords: Change problems, combination, comparison, equalization.

\section{Introducción}

La investigación surge a raíz de la experiencia en las aulas de la IEP $\mathrm{N}^{\circ}$ 71001 Almirante Miguel Grau de Puno, al notar que los niños y niñas de cuarto grado tenían dificultades en la resolución de problemas aritméticos de enunciado verbal (PAEV), los resultados de la Evaluación Censal de Estudiantes ECE 2016 a nivel de la región Puno, que muestra que sólo un $27,4 \%$ de estudiantes de cuarto grado están en el nivel satisfactorio, que es el nivel de logro esperado en el uso de números $\mathrm{y}$ resolución de problemas, el 42,6\% se encuentran en proceso, el $21,5 \%$ en inicio y un 8,5\% están por debajo del nivel de inicio. Así mismo a nivel de la UGEL Puno los resultados de la ECE 2016 fueron los siguientes: el 35,3\% se encuentran en el nivel satisfactorio, el
$41,2 \%$ se encuentran en proceso el 17,5\% está en el nivel de inicio y el 6,0\% se encuentran previo al inicio, lo cual indica que ni siquiera responden las preguntas más sencillas.

Frente a esta problemática consideramos necesario aplicar el método de Polya como estrategia metodológica para resolver problemas aritméticos de enunciado verbal (PAEV) para erradicar la concepción de la matemática como un área aburrida y difícil. Se debe tomar conciencia acerca de la problemática vivida en torno a este tema, pero también es pertinente tomar las medidas adecuadas para lograr mejores niveles de aprendizajes.

\section{Estado de la cuestión.}

Badillo, P., Salazar, A. \& Vera, G. (2012), persiguieron el objetivo de establecer la efectividad del programa 
Volumen 1, Número 2, Mayo-Agosto 2021 Fecha de publicación: 10-05-2021 - Fecha de aceptación:20-04-2021

\section{GnosisWisdam}

"GPA-RESOL" en el incremento del las Instituciones Educativas Públicas del nivel de logro en la resolución de problemas aritméticos aditivos $\mathrm{y}$ Concejo Educativo Municipal de la Molina en el año 2011, concluye que:, sustractivos en estudiantes de segúndo grado de primaria de dos instituciones educativas una de gestión estatal y otra privada del distrito de San Luis. Concluye que: El nivel de logro en resolución de problemas aritméticos aditivos y sustractivos en estudiantes de segundo grado de primaria de dos instituciones educativas una de gestión estatal y otra particular del distrito de San Luis después de la aplicación del programa GPA - RESOL es altamente significativo. En el momento del pre test el grupo experimental difiere del grupo control y al interior de los grupos, los estudiantes de la institución de gestión privada evidencian un mejor nivel de logro en la resolución de problemas aritméticos aditivos y sustractivos. En el momento post test el grupo experimental tiene mayor nivel, pero al interior del grupo experimental el tipo de gestión no evidenció mayor impacto en el nivel de logro en la resolución de problemas aritméticos aditivos y sustractivos.

Bastiand (2012), tuvo como objetivo determinar la relación que existe entre la comprensión lectora y la resolución de problemas matemáticos en los estudiantes de sexto grado de primaria de los alumnos se ubican de la siguiente manera: Comprensión: En proceso, con una nota de 11.2. Planificación: Logro previsto, con una nota de 12.6. Ejecución: En inicio, con una nota de 09.2. Comprobación: En inicio, con una nota de 08. El 55\% de los alumnos de la muestra resolvieron correctamente las preguntas de la prueba de resolución de problemas matemáticos, de los cuales, el $56 \%$ resolvieron correctamente las preguntas de comprensión; el 63\%, las preguntas de planificación; el $45 \%$ las preguntas de ejecución, y el 39\%, las preguntas de comprobación.

Polya (1971), propone metódicamente cuatro pasos para resolver un problema de acuerdo a la necesidad del alumno y del docente de adquirir un trabajo personal del educando, en donde el maestro debe ayudarle pero no demasiado, aunque es cierto que resolver un problema también depende del estadio mental del individuo porque un problema para un niño entre 5 y 6 años, es un ejercicio aritmético para un adolescente entre 12 y 25 años de edad, la concepción de esta propuesta está basada en el pensamiento de autores como Piaget (1967) que consideran la 


\section{GnasisWisdam}

importancia de la estructura física del cerebro humano, dentro de unas condiciones médicamente normales.

a) Entender el problema

b) Configurar un plan

c) Ejecutar el plan

d) Mirar hacia atrás

Martínez (1986) plantean: "Que la enseñanza problémica concibe el conocimiento como un proceso en el cual se desarrollan formas de pensamiento" (p. 20). Es decir, formas de realidad, y en el que interviene y se desarrolla la creatividad. "la resolución de problemas es identificada como una actividad crucial en las ciencias, además de ser inherente en la vida diaria" es "un proceso prioritario para el desarrollo en los estudiantes de habilidades operacionales formales, el pensamiento proporcional y el pensamiento lógicodeductivo". (Tobón, 2006, p. 23).

En el campo de la didáctica de las ciencias, García (1983) plantea "Que la resolución de problemas ha sido estudiada desde diferentes enfoques, como estrategia para generar cambios conceptuales, metodológicos y actitudinales y para superar la metodología del sentido común". (p. 24); Gil Pérez (1986) la propone como "la capacidad relacionada con la organización y estructuración de la información en la mente". (p. 28); Palacios (1992) como "proceso que puede ser enseñado a los novatos a partir del estudio de la forma en que resuelven problemas los expertos" (p. 30).

MINEDU (2013) menciona que los problemas aritméticos de enunciado verbal (PAEV) son: Problemas Aditivos (en los que se requiere sumar y restar) y Problemas multiplicativos (en los que se requiere multiplicar y dividir).

\section{- Problemas aritméticos aditivos}

De acuerdo con Puig y Cerdán (1988), los problemas aritméticos aditivos, se clasifican en cuatro categorías:

a) Problemas de combinación

b) Problemas de cambio

c) Problemas de igualación

d) Problemas de comparación

\section{- Problemas}

aritméticos

\section{multiplicativos}

Maza (1991) hay un cierto acuerdo en dividir los problemas de estructura multiplicativa en los siguientes tipos:
a) De reparto equitativo
b) De factor $\mathrm{N}$ o comparación multiplicativa
c) De razón o de tasa
d) De producto cartesiano

El enunciado general del problema es: ¿Cuál es el nivel de logro de resolución de problemas aritméticos de enunciado 
Volumen 1, Número 2, Mayo-Agosto 2021 Fecha de publicación: 10-05-2021 - Fecha de aceptación:20-04-2021

\section{GnasisWisdam}

verbal con la aplicación del Método

Polya como estrategia en estudiantes de cuarto grado de la I.E.P. $\mathrm{N}^{\circ} 71001$ Almirante Miguel Grau de Puno?; de manera que el propósito es determinar el nivel de logro de la resolución de problemas aritméticos de enunciado verbal aplicando el Método Polya como estrategia en estudiantes de cuarto grado de la I.E.P. $N^{\circ} 71001$ Almirante Miguel Grau de Puno. La hipótesis que responde a esta interrogante es que la aplicación del método de Polya utilizada como estrategia permite el nivel de logro significativo en la resolución de problemas aritméticos de enunciado verbal en estudiantes de cuarto grado.

\section{Metodología de investigación}

La población está constituida por los alumnos de cuarto grado de primaria de la IEP No 71001 "Almirante Miguel Grau" de Puno, con un total de 198 estudiantes, distribuida en 6 secciones. Se ha seleccionado la muestra en forma intencionada, mediante el criterio de azar simple por la que la sección de cuarto "C" recae ser grupo de control y la sección "F" como grupo experimental; en consecuencia la muestra estará conformada por 64 estudiantes que se especifican en la siguiente tabla:

\section{Tabla 1}

Niños y niñas de cuarto grado de la IEP $N^{\circ} 71001$ “Almirante Miguel Grau” que conforman la muestra - Puno - 2018.

\begin{tabular}{ccc}
\hline GRUPO & SECCIÓN & $\begin{array}{c}\mathbf{N}^{\circ} \text { DE } \\
\text { ALUMNO } \\
\text { S POR } \\
\text { SECCIÓN }\end{array}$ \\
\hline Grupo & F & 32 \\
Experimenta & & \\
1 & & \\
Grupo de & $\mathrm{C}$ & 32 \\
Control & $\mathbf{2 1}$ & $\mathbf{6 4}$ \\
TOTAL & & \\
\hline
\end{tabular}

Fuente: Nómina de matriculados IEP $N^{\circ}$ 71001 Puno - 2018

\section{Tipo y diseño de investigación.}

La investigación es de tipo experimental, mientras que el diseño de investigación correspondiente al cuasi experimental, con prueba de entrada (pre test) y prueba de salida (post test) en grupos intactos, un grupo de control y otro grupo experimental.

\section{Técnicas e instrumentos de investigación.}

La técnica utilizada fue el examen d y como instrumento se ha aplicado una prueba de entrada (pre prueba) y prueba de salida (post prueba), sobre resolución de problemas aritméticos de enunciado verbal. Los métodos experimentales son los adecuados para poner a prueba este tipo de instrumentos.

Procedimiento de la investigación. 


\section{GnasisWisdam}

Para concretar la investigación se procedió de la siguiente manera:

a. Se solicitó al Director de la Institución Educativa Primaria $\mathrm{N}^{\circ}$ 71001 Almirante Miguel Grau de la ciudad de Puno, para desarrollar las sesiones de aprendizaje aplicando el Método Polya como estrategia en la resolución de problemas aritméticos de enunciado verbal.

b. Aplicación de las pruebas de entrada (pre test) a ambos grupos (experimental y control), para obtener información sobre el nivel de logro en la resolución de problemas aditivos $\mathrm{y}$ multiplicativos previo al tratamiento experimental.

c. Desarrollo de actividades de aprendizaje sobre resolución de problemas aritméticos de enunciado verbal, en este momento se planean y se desarrollan dichos problemas con el grupo experimental teniendo en cuenta el método Polya, enfatizando en la resolución de problemas y utilizando material didáctico adecuado para facilitar el aprendizaje. Se evalúan las capacidades y desempeños de los estudiantes. d. Al concluir el desarrollo de las actividades se procedió con la aplicación de la prueba de salida o pos test a ambos grupos para determinar el nivel de logro en la resolución de problemas aritméticos de enunciado verbal.

e. Procedimiento de los datos recolectados para su análisis e interpretación, para lo cual se siguió los siguientes pasos: Elaboración de tablas y figuras estadísticas del grupo experimental y del grupo control, basándose en las pruebas de entrada (pre test) y salida (pos test). Aquí, se analizaron los datos obtenidos en relación a las variables de estudio.

\section{Análisis Estadístico.}

Para el análisis estadístico, se ha utilizado la estadística descriptiva para el procesamiento de datos, dentro de ello fue necesario la media aritmética, la varianza y para poder probar las hipótesis se aplicaron la distribución de diferencia de medias Zeta calculada.

\section{Resultados:}

Resultados obtenidos en la pre prueba Tabla 2. 
Volumen 1, Número 2, Mayo-Agosto 2021 Fecha de publicación: 10-05-2021 - Fecha de aceptación:20-04-2021

\section{GnasisWisdam}

Resultados de la pre prueba sobre resolución de problemas aritméticos en estudiantes de la IEP $N^{\circ} 71001$ “Almirante Miguel Grau” - Puno - 2018

\begin{tabular}{|c|c|c|c|c|c|}
\hline & & \multicolumn{4}{|c|}{ GRUPOS } \\
\hline & & \multicolumn{2}{|c|}{ CONTROL } & \multicolumn{2}{|c|}{ EXPERIMENTAL } \\
\hline & & f & $\%$ & f & $\%$ \\
\hline $\begin{array}{l}\text { LOGRO } \\
\text { DESTAC. }\end{array}$ & AD & 0 & 0.00 & 0 & 0.00 \\
\hline $\begin{array}{l}\text { LOGRO } \\
\text { PREV. }\end{array}$ & $\mathbf{A}$ & 7 & 21.88 & 6 & 18.75 \\
\hline $\begin{array}{l}\text { EN } \\
\text { PROCESO }\end{array}$ & B & 12 & 37.50 & 12 & 37.50 \\
\hline EN INICIO & C & 13 & 40.63 & 14 & 43.75 \\
\hline \multicolumn{2}{|c|}{ TOTAL } & 32 & 100 & 32 & 100 \\
\hline
\end{tabular}

Fuente: Resultados de la pre prueba.

En la tabla 2, se puede apreciar la información en donde los estudiantes del grupo control y del grupo experimental no superan los bajos niveles de logro de resolución de problemas aritméticos de enunciado verbal al aplicarles una pre prueba. Los cálculos estadísticos de la Media Aritmética: grupo Control $\bar{X}_{\mathrm{C}}=$ 10,21 y grupo Experimental : $\bar{X} \mathrm{e}=$ 10,03 y de la Varianza: grupo Control: $\mathrm{S}^{2} \mathrm{c}=7,98$ y grupo Experimental $\mathrm{S}^{2} \mathrm{e}=$ 7,16 , indican que los estudiantes de ambos grupos, se encuentran en niveles equivalentes, ambos en el nivel de inicio.

Resultados obtenidos en el proceso del experimento

Tabla 3

Proceso de resolución de problemas aditivos de enunciado verbal por los estudiantes de la IEP $N^{o} 71001$ “Almirante Miguel Grau” Puno-2018

\begin{tabular}{|c|c|c|c|c|c|c|c|c|c|}
\hline \multirow{2}{*}{\multicolumn{2}{|c|}{$\begin{array}{c}\text { NIVEL DE } \\
\text { LOGRO }\end{array}$}} & \multicolumn{8}{|c|}{ RESOLUCIÓN DE PROBLEMAS ADITIVOS } \\
\hline & & \multicolumn{2}{|c|}{$\begin{array}{l}\text { COMBIN } \\
\text { ACIÓN }\end{array}$} & \multicolumn{2}{|c|}{$\begin{array}{l}\text { CAMB } \\
\text { IO }\end{array}$} & \multicolumn{2}{|c|}{$\begin{array}{l}\text { COMPAR } \\
\text { ACIÓN }\end{array}$} & \multicolumn{2}{|c|}{$\begin{array}{l}\text { IGUALA } \\
\text { CIÓN }\end{array}$} \\
\hline & & f & $\%$ & f & $\%$ & f & $\%$ & f & $\%$ \\
\hline LOGR & A & & & & 15 & & 12.5 & & \\
\hline $\mathrm{O}$ & D & 3 & 9.3 & 5 & .6 & 4 & 0 & 3 & 9.3 \\
\hline DEST & & & 8 & & 3 & & & & 8 \\
\hline \multicolumn{10}{|l|}{$\mathrm{ACA}$} \\
\hline \multicolumn{10}{|l|}{ DO } \\
\hline LOGR & $\mathbf{A}$ & 1 & 43. & 1 & 31 & & 28.1 & 1 & 37. \\
\hline $\mathrm{O}$ & & 4 & 75 & 0 & .2 & 9 & 3 & 2 & 50 \\
\hline PREV & & & & & 5 & & & & \\
\hline \multicolumn{10}{|l|}{ ISTO } \\
\hline EN & B & 1 & 31. & 1 & 43 & 1 & 50.0 & 1 & 43. \\
\hline PROC & & 0 & 25 & 4 & .7 & 6 & 0 & 4 & 75 \\
\hline ESO & & & & & 5 & & & & \\
\hline EN & C & & 15. & & & & & & \\
\hline INICI & & 5 & 63 & 3 & 9. & 3 & 9.38 & 3 & 9.3 \\
\hline $\mathrm{O}$ & & & & & 38 & & & & 8 \\
\hline \multirow{2}{*}{\multicolumn{2}{|c|}{ TOTAL }} & 3 & 100 & 3 & 10 & 3 & 100 & 3 & 10 \\
\hline & & 2 & & 2 & 0 & 2 & & 2 & 0 \\
\hline
\end{tabular}

Fuente: Registro de notas de evaluación de proceso.

En la tabla 3, los indicadores de resolución de problemas aditivos en promedio indican que: el $11,72 \%$ lograron alcanzar el nivel de logro destacado, el 35,16\% el nivel de logro previsto; el 42,19\% el nivel en proceso y finalmente el 10,94\% continúan en el nivel en inicio.

En consecuencia se puede deducir que los estudiantes del grupo experimental, superaron los bajos niveles de resolución de problemas aritméticos aditivos de enunciado verbal en el proceso experimental.

Tabla 4 


\section{GnasisWisdam}

Proceso de resolución de problemas multiplicativos de enunciado verbal por los estudiantes de la IEP $N^{o} 71001$ “Almirante Miguel Grau” Puno - 2018 Fuente: Registro de notas de evaluación de proceso

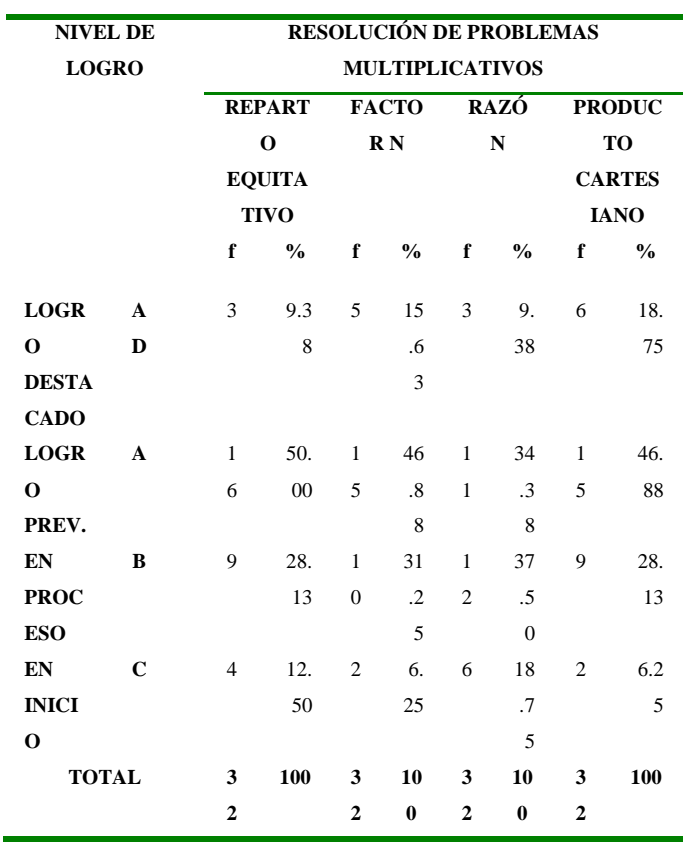

En la tabla 4, se puede apreciar la información sobre los indicadores de resolución de problemas multiplicativos en promedio se observa que: el 13,29\% lograron alcanzar el nivel de logro destacado, el 44,54 el nivel de logro previsto; el 31,25\% el nivel en proceso y finalmente el 10,94\% continúan en el nivel en inicio. En consecuencia se puede deducir que los estudiantes del grupo experimental, superaron los bajos niveles de resolución de problemas aritméticos multiplicativos de enunciado verbal en el proceso experimental.

\section{Resultados obtenidos en la post prueba}

$\mathrm{Al}$ igual que en la prueba de entrada o pre prueba, para poder responder a los ítems de la post prueba o prueba de salida, los niños manifestaban sus habilidades para resolver las situaciones planteadas referentes problemas aritméticos de enunciado verbal, los cuales fueron procesados $\mathrm{y}$ presentados en tablas $\mathrm{y}$ figuras siguientes:

Tabla 5

Resultados de la post prueba sobre resolución de problemas aritméticos en estudiantes de la IEP $N^{o} 71001$ “Almirante Miguel Grau” Puno-2018

\begin{tabular}{lccccc}
\hline & & \multicolumn{5}{c}{ GRUPOS } \\
\cline { 3 - 6 } \multicolumn{1}{c}{ NIVEL DE LOGRO } & \multicolumn{2}{c}{ CONTROL } & EXPERIMENTAL \\
& & f & $\%$ & f & $\%$ \\
& & & & & \\
LOGRO & AD & 4 & 12.50 & 10 & 31.25 \\
$\begin{array}{l}\text { DESTAC. } \\
\text { LOGRO }\end{array}$ & A & 11 & 34.38 & 15 & 46.88 \\
$\begin{array}{l}\text { PREV. } \\
\text { EN }\end{array}$ & B & 12 & 37.50 & 6 & 18.75 \\
$\begin{array}{l}\text { PROCESO } \\
\text { EN INICIO }\end{array}$ & C & 5 & 15.63 & 1 & 3.13 \\
\multicolumn{1}{c}{ TOTAL } & $\mathbf{3 2}$ & $\mathbf{1 0 0}$ & $\mathbf{3 2}$ & $\mathbf{1 0 0}$ \\
\hline
\end{tabular}

Fuente: Resultados de la post prueba

En la tabla 5, se puede apreciar la información concerniente a los resultados de la pos prueba sobre resolución de problemas aritméticos de enunciado verbal en donde los cálculos estadísticos de la media aritmética para el grupo de Control $\bar{X}$ c $=12,39 \mathrm{y}$ Experimental $\bar{X}$ e $=14,89$ y la varianza 


\section{GnosisWisdam}

para el grupo de control $\mathrm{S}^{2} \mathrm{c}=5,12 \mathrm{y}$

$$
\text { - } \mathrm{Xe} \neq \mathrm{Xc}
$$

Experimental $\mathrm{S}^{2} \mathrm{e}=4,32$, indican que, los estudiantes del grupo experimental superan a los del grupo de control en puntaje promedio ubicándose en el nivel de logro previsto; mientras que los estudiantes del grupo de control se ubican en el nivel en proceso. Esta información permite deducir que el método Polya como estrategia dio efectos significativos en el nivel de resolución de problemas aditivos de enunciado verbal.

Para poder comprobar las hipótesis, se ha utilizado la distribución $Z_{c}$ Zeta calculada con un nivel de significancia del $95 \%$, es decir $\alpha=0.05$, cuyo valor en la tabla responde a $\mathrm{Zc}= \pm 1,96$ se plantean las siguientes hipótesis estadísticas:

- Ho: El promedio de las notas obtenidas en el pre test por los estudiantes del grupo experimental es igual a los obtenidos por el grupo control.

$$
\text { - } \mathrm{Xe}=\mathrm{Xc}
$$

- Ha: El promedio de las notas obtenidas en el pre test por los estudiantes del grupo experimental es diferente a los obtenidos por el grupo control.

Aplicando la prueba estadística de diferencia de medias se obtiene: $Z_{c}=4,6$ que permite rechazar la hipótesis nula, en consecuencia, decidimos aceptar a la hipótesis alterna $\mathrm{Ha}$,: El promedio de las notas obtenidas en la pos prueba por los estudiantes del grupo experimental es mayor a los obtenidos por el grupo control.

\section{Discusión de resultados}

Tomando en cuenta a Tobón (2006), la resolución de problemas es una actividad inherente a la vida diaria prioritaria para el desarrollo en los estudiantes de habilidades operacionales formales, el pensamiento proporcional y el pensamiento lógico-deductivo. Desde ese punto de vista, los logros obtenidos en el proceso de ejecución del experimento en cuanto se refiere a los indicadores de resolución de problemas referidos a combinación: el 15,63\% se ubicaron en el nivel en inicio; el 31,25\% se encuentran en el nivel en proceso, el $43,75 \%$ se encuentran en el nivel de logro previsto y el 9,38\% se ubican en el nivel de logro destacado. Al respecto Polya (1971) plantea el hecho de resolver problemas como un proceso metódico y procedimental en el que el alumno utiliza 
Volumen 1, Número 2, Mayo-Agosto 2021 Fecha de publicación: 10-05-2021 - Fecha de aceptación:20-04-2021

\section{GnasisWisdam}

su razonamiento en la búsqueda de una solución a una situación problemática.

En los indicadores de resolución de problemas referidos a cambio en la presente investigación: el 9,38\% se ubicaron en el nivel en inicio; el 43,75\% se encuentran en el nivel en proceso, el $31,25 \%$ se encuentran en el nivel previsto y el $15,63 \%$ se ubican en el nivel logro destacado; en los indicadores de resolución de problemas referidos a comparación: el 9,38\% se ubicaron en el nivel en inicio; el 50,00\% se encuentran en el nivel en proceso, el $28,13 \%$ se encuentran en el nivel previsto y el $12,50 \%$ se ubican en el nivel destacado; como también en los indicadores de resolución de problemas referidos a igualación: el 9,38\% se ubicaron en el nivel en inicio; el 43,75\% se encuentran en el nivel en proceso, el $37,50 \%$ se encuentran en el nivel previsto y el 9,38\% se ubican en el nivel destacado. Estos resultados permitieron deducir que los niños y niñas del grupo experimental, superaron los bajos niveles de resolución de problemas aritméticos aditivos de enunciado verbal en el proceso experimental coincidentemente Calvo (2008) descubrió que las dificultades de los estudiantes de sexto grado de educación primaria para la resolución de problemas matemáticos, no radican en el estudiante mismo, sino que entran otros aspectos en juego, tales como la metodología empleada por el docente o la actitud que éste tenga hacia la materia. Cardona (2008) evidenció que recurriendo a la aritmética los alumnos daban paso al álgebra con mayor seguridad, la estrategia de resolución de problemas resultó ser adecuada para iniciar en los estudiantes el código algebraico a través de su uso los conceptos algebraicos que se desarrollaron por necesidad. En este mismo sentido los ítems 1,2 y 3 de esta investigación permitieron que el niño ha identificado los datos y la incógnita esto significa que el niño ha comprendido el problema, luego platea realizar una operación de multiplicación esto constituye la segunda fase de realizar un plan y luego realiza la operación, esto significa que ha ejecutado el plan en la tercera fase $y$ finalmente no ha comprobado, pero si ha resuelto correctamente. En el ítem 4 el niño ha identificado los datos y la incógnita esto significa que el niño ha comprendido el problema, luego no ha plateado bien realizar una operación por lo que no ha resuelto correctamente el problema. Frente a este resultado, Carrero (2006) manifiesta que la planificación va inmersa a las estrategias, las cuales 
Volumen 1, Número 2, Mayo-Agosto 2021 Fecha de publicación: 10-05-2021 - Fecha de aceptación:20-04-2021

\section{GnosisWisdam}

deben ser adecuadas para que los alumnos puedan construir su propio aprendizaje tomando en cuenta sus experiencias y necesidades previas.

En los indicadores de resolución de problemas referidos a reparto equitativo de esta investigación: el 12,50\% se ubicaron en el nivel en inicio; el 28,13\% se encuentran en el nivel en proceso, el $50 \%$ se encuentran en el nivel previsto y el 9,38\% se ubican en el nivel destacado; En los indicadores de resolución de problemas referidos a factor " $n$ ": el $6,25 \%$ se ubicaron en el nivel en inicio; el $31,25 \%$ se encuentran en el nivel en proceso, el $46,88 \%$ se encuentran en el nivel previsto y el $15,63 \%$ se ubican en el nivel destacado. Así mismo en los indicadores de resolución de problemas referidos a razón: el 18,75\% se ubicaron en el nivel en inicio; el $37,50 \%$ se encuentran en el nivel proceso, el $34,38 \%$ se encuentran en el nivel previsto y el 9,38\% se ubican en el nivel destacado.; finalmente en los indicadores de resolución de problemas referidos a producto cartesiano: el $6,25 \%$ se ubicaron en el nivel inicio; el $28,13 \%$ se encuentran en el nivel proceso, el $46,88 \%$ se encuentran en el nivel previsto y el $18,75 \%$ se ubican en el nivel destacado. En este caso en el ítem 9 y 10 el niño ha identificado los datos y la incógnita esto significa que el niño ha comprendido el problema, luego platea realizar una operación de división esto constituye la segunda fase de realizar un plan y luego realiza la operación al tanteo, esto significa que ha ejecutado el plan en la tercera fase y finalmente no ha comprobado, pero si ha resuelto correctamente al respecto Carrero (2006) continua manifestando que la p'lanificaci9on de la resolución de problemas y hacer una plan de solución son aspectos muy importantes para la solución de un problema matemático. Estos resultados permitieron deducir que los niños y niñas del grupo experimental, superaron los bajos niveles de resolución de problemas aritméticos multiplicativos de enunciado verbal en el proceso experimental. A este respecto Astola, Salvador y Vera (2012) manifiestan que el nivel de logro en resolución de problemas aritméticos aditivos $\mathrm{y}$ sustractivos en estudiantes de segundo grado de primaria es altamente significativo, los cuales coinciden con los resultados encontrados.

Los resultados de la post prueba sobre resolución de problemas aritméticos de enunciado verbal indican que en el grupo control el $12.50 \%$ y en el grupo experimental el $31.25 \%$ lograron el nivel destacado. Asimismo en el grupo pág. 23 
Volumen 1, Número 2, Mayo-Agosto 2021 Fecha de publicación: 10-05-2021 - Fecha de aceptación:20-04-2021

\section{GnasisWisdam}

control; el $34.38 \%$ se encuentran en el nivel de logro previsto y en el grupo experimental el $46.88 \%$ se encuentran en el nivel de logro previsto. Yáñez (2010) encontró que las experiencias de aprendizaje en el aula permitieron la resolución de problemas, partiendo de las experiencias consensuadas entre el participante, el docente y las estrategias utilizadas en la instrucción; que justamente si observamos las medias aritméticas obtenidas en la prueba de salida de esta investigación, de 12,39 puntos en el grupo de control y 14,89 en el grupo experimental los cuales indican los logros obtenidos los cuales indicar que se ha superado los bajos niveles de logro de resolución de problemas aritméticos de enunciado verbal al aplicarles una post prueba.

Los resultados generales en la post prueba indican que en el grupo de control, el $15,63 \%$ se encuentran en el nivel inicio, el 37,50\% se encuentran en el nivel proceso y el $34,38 \%$ se encuentran en el nivel de logro previsto y solamente el 12,50\% llegaron al nivel destacado; mientras que en el grupo experimental, el 3,13\% se encuentran en el nivel inicio, el 18,75\% se encuentran en el nivel proceso, el $46,88 \%$ se encuentran en el nivel previsto y el $31,25 \%$ llegaron al nivel destacado.
Estos datos indican que los niños y niñas del grupo experimental lograron mejores niveles de aprendizaje en la resolución de problemas. Estos resultados también superaron a los resultados encontrados por Bastiand (2012) en donde los estudiantes de sexto grado de primaria en la prueba de resolución de problemas matemáticos, se ubican en el nivel "en proceso" con una nota desaprobatoria de 11 puntos. En las fases de la resolución de problemas matemáticos, los alumnos se ubican de la siguiente manera: Comprensión: En proceso, con una nota de 11.2. Planificación: Logro previsto, con una nota de 12.6. Ejecución: En inicio, con una nota de 09.2. al respecto Cardona (2008) en su estudio de tipo cuasi-experimental a partir del Método Polya para facilitar la resolución de problemas matemáticos, encontró que para la solución de cualquier problema, es requisito principal la comprensión lectora.

\section{Conclusiones.}

El Método Polya, permite alcanzar mejores niveles de logro en la resolución de problemas aritméticos de enunciado verbal en los estudiantes de cuarto grado de la Institución Educativa Primaria $\mathrm{N}^{\mathrm{o}}$ 71001 "Miguel Grau" de la ciudad de Puno en el año 2017, porque este método les permite desarrollar habilidades de 


\section{Gnasis Wisdam}

resolución de problemas aditivos y multiplicativos, esta conclusión es evidenciada por la media aritmética del grupo experimental de 14,89 puntos y la prueba de hipótesis de 4,6 de la distribución de $\mathrm{Z}$ calculada de diferencia de medias.

El nivel de logro en la resolución de problemas aritméticos aditivos de enunciado verbal, está en el nivel de proceso y logro previsto aplicando el método de George Polya en el que $42,19 \%$ de estudiantes se ubicaron en el nivel de proceso y el $35,16 \%$ en el nivel de logro previsto y el $11,71 \%$ en el nivel de logro destacado; sin embargo el 10,94\% aún se encuentran en el nivel de inicio.

El nivel de logro en la resolución de problemas aritméticos multiplicativos de enunciado verbal, está en proceso y logro previsto aplicando el método de George Polya en el que $31,25 \%$ de estudiantes se ubicaron en el nivel de proceso y el $44,53 \%$ en el nivel de logro previsto y el 13,28\% en el nivel de logro destacado; sin embargo el 10,94\% aún se encuentran en el nivel de inicio.

\section{Referencias:}

Astola Badillo, P. C., Salvador Carrillo, A. E. y Vera Pacco, G. (2012). Efectividad del programa "GPARESOL" en el incremento del nivel de logro en la resolución de problemas aritméticos aditivos $\mathrm{y}$ sustractivos en estudiantes de segundo grado de primaria de dos instituciones educativas, una de gestión estatal y otra privada del distrito de San Luis. (Tesis de maestría). Pontificia Universidad Católica del Perú, Lima, Perú.

Ausubel, N. (1983) Psicología Educativa: Un punto de vista cognoscitivo ( $2^{\mathrm{a}}$ ed.) México: Trillas.

Bastiand Valverde, M. A. (2012). Relación entre comprensión lectora y resolución de problemas matemáticos en estudiantes de sexto grado de primaria de las instituciones educativas públicas del Concejo Educativo Municipal de La Molina - 2011. (Tesis de maestría). Universidad Nacional Mayor de San Marcos, Lima, Perú.

Betancourt, J. (2007). Planificación de juegos lúdicos como estrategia para mejorar la Enseñanza y el aprendizaje de la matemática. (Tesis de Maestría). Universidad Valle del Momboy, Valera, Venezuela.

Calvo, M. (2008). Enseñanza eficaz de la resolución de problemas en 


\section{GnosisWisdam}

matemáticas. Revista Educación, 32(1), 123-138. ISSN: 03797082, 2008.

Cardona, M. (2008). Método Polya en el diseño de estrategias para facilitar la resolución de problemas relacionados con áreas de figuras planas. (Tesis de maestría). Universidad Pedagógica Nacional Francisco Morazán, San Pedro Sula, Honduras.

Carrero, L. (2006). Planificación de estrategias didácticas para la enseñanza de la Matemática en los alumnos del 4to Grado de Educación Básica. (Tesis de Maestría). Universidad Valle del Momboy, Valera, Venezuela.

Carrillo, J. (1998). La resolución de problemas en la enseñanza secundaria. Ejemplificaciones del para qué. Épsilon, 40, 15-16.

Cerdán, F y Puig L. (1995). Problemas aritméticos escolares. (2da ed.) Madrid: Síntesis S.A.

Chávez, G. (2013). Método Polya. El pensamiento del estratega. México: Plaza y Valdés, S.A. de C.V.

Descartes, R. (1993). Discurso del método.

Meditaciones metafísicas. (M. García Morente ed.). Madrid: Espasa Calpe.

García, E. (1983). Evolución de la didáctica de las matemáticas como disciplina científica.

Gil Pérez, D. (1986). La enseñanza de las ciencias y la matemática. España: Popular.

Leibniz, G. W. (1986). Discurso de metafísica. (J. Marías ed.). Madrid: Alianza Editorial.

Majimutov, M. I. (1983). La enseñanza problémica. La Habana: Editorial Pueblo y Educación, Ministerio de Educación.

Martínez Llantada, M. (1986). Categorías principios y métodos de la enseñanza problémica. La Habana: Editorial Pueblo y Educación.

Mayer, R. (1993). Resolución de problemas y cognición. Barcelona: Paidós.

Ministerio de Educación. (2013). Rutas de Aprendizaje - Área Curricular de Matemática. Lima, Perú.

Ortiz, A. (1986). Metodología de la Enseñanza Problémica en el aula de clase. Colombia: Asiesca Ediciones

Palacios, C. y López Rupéres, F. (1992). Resolución de problemas de química, mapas conceptuales y pág. 26 
Volumen 1, Número 2, Mayo-Agosto 2021 Fecha de publicación: 10-05-2021 - Fecha de aceptación:20-04-2021

\section{GnosisWisdam}

estilo cognoscitivo. Revista de Educación, 293-314.

Piaget, J, (1967). Programas y métodos de la epistemología genética. En J. Piaget, Psicología, lógica y comunicación (N. Bastard, Trad.) Buenos Aires: Ediciones Nueva Visión: Colección Ensayos. (Trabajo realizado en el Centro Internacional de Epistemología Genética durante el año 195556).

Polya, G. (1971). Cómo plantear y resolver problemas. México: Trillas.

Puig, L. (1999). Problemas aritméticos escolares. Madrid: Síntesis.

Puig, L. y Cerdán, F. (1988). Problemas aritméticos escolares. Madrid, España: Síntesis.

Rodríguez Quintana, E. (2005). Metacognición, resolución de problemas y enseñanza de las matemáticas. Una propuesta integradora desde el enfoque antropológico. (Tesis de doctorado). Universidad Complustense, Madrid, España.

Tobón, S. (2006). Competencia, calidad y educación superior. Lima: Editorial Magisterio. Página de créditos.
Vergnaud, G. (1990). La théorie des champs conceptuels. Recherches en didactique des mathématiques, 10, 133 -170.

Vergnaud, G. (1995). El niño, las matemáticas y la realidad, problema de las matemáticas en la escuela. Trillas: México.

Vygotski, L. S. (1989). El desarrollo de los procesos psicológicos superiores (S. Furió, Trad. de la versión en inglés "Mind in Society. The Development of Higher Psychological Processes"). Barcelona: CríticaGrijalbo (Trabajo publicado póstumamente en 1935).

Yánez Bolívar, T. M. (2010). Efectos de la Resolución de Problemas Mediado por el Weblog sobre el Rendimiento en Matemática (Tesis de maestría). Universidad Central de Venezuela, Venezuela.

\section{Correspondencia}

Lino, Vilca Mamani

lvilcam@unap.edu.pe 